MNE Paper: S. Hosaka et al.

\title{
Ordering of 6-nm-sized nanodot arrays with 10-nm-pitch using self-assembled block copolymers along electron beam-drawn guide-lines
}

\author{
Sumio Hosaka ${ }^{1 *}$, Takashi Akahane ${ }^{1}$, Miftakhul Huda ${ }^{1}$, Takuya Komori ${ }^{1}$, Hui Zhang ${ }^{1}$ \\ and You Yin', \\ ${ }^{1}$ Graduate School of Science and Technology, Gunma University, \\ 1-5-1 Tenjin-cho, Kiryu 376-8515, Japan \\ *Corresponding author: hosaka@gunma-u.ac.jp; Tel. +81-277-30-1721; Fax. +81-277-30-1707
}

\begin{abstract}
We studied the possibility to form long-rangeordering of self-assembled nanodot arrays with a dot size of $6 \mathrm{~nm}$ and a dot pitch of $12 \mathrm{~nm}$ using guide-line templates for ultrahigh-density patterned media. This was realized by using self-assembled block copolymers of polystyrenepoly dimethyl siloxane (PS-PDMS) and the templates made of hydrogen silsesquioxane (HSQ) negative electron beam (EB) resist. We have demonstrated that the method could possibly achieve a minimum pitch of about $10 \mathrm{~nm}$ using PS-PDMS of molecular weight 7000$1500 \mathrm{~g} / \mathrm{mol}$ and EB-drawn guide lines. These results evidence the promising potential of a technology for achieving $5 \mathrm{Tbit} / \mathrm{in}^{2}$ magnetic storage devices.
\end{abstract}

Keywords: Nanodot, self-assembly, electron-beam drawing, graphoepitaxy, patterned media, magnetic recording.

\section{Introduction}

Nanoscale devices and ultrahigh-density storage media necessitate a fabrication technology for ultrahigh-packed dot arrays. Currently, self-assembly of such nanodevices and media is gaining great interest as a promising method to form fine patterns and achieve high throughput packing of fine dot arrays with a pitch of $<20 \mathrm{~nm}$ in a large area [1-3]. However, self-assembly has some technical disadvantages such as disordering and defects. Bita et al. addressed this issue by proposing the graphoepitaxy of self-assembled block copolymers (BCPs) using two-dimensional periodic-patterned templates [1]. Nevertheless, the template pattern requires a good arrangement of the guide post pitch with a deviation of $<5 \mathrm{~nm}$. Controlling this value is very critical for practical application. Hence, it is vital to develop a practical method where the deviation has a larger margin. 
On the other hand, Sakurai et al. [4], Black [5], and Kitano et al. [6] proposed an alternative guide-line template for self-assembly. They applied optical lithography to fabricate the guide-line template for use in graphoepitaxy. Furthermore, they demonstrated that the proposed technology could be applied to form nanodot arrays using self-assembly method. The number of ordered nanodot arrays between the lines could be 4-7. The arrangement was done using self-assembled BCP with a pitch larger than $30 \mathrm{~nm}$. Recently, our group demonstrated the long-range ordering of self-assembled poly dimethyl siloxane (PDMS) dot arrays with a pitch of $33 \mathrm{~nm}$ [7]. However, these experiments did not prove that the method could be used for patterned media with a density of more than $5 \mathrm{~Tb} / \mathrm{in}^{2}$. In order to realize this objective, we conducted research on ordering nanodot arrays with pitch of $\sim 10 \mathrm{~nm}$ using polystyrene-PDMS (PS-PDMS) self-assembly method with electron beam (EB)-drawn guide pattern.

Therefore, in this paper, we have described the selection of PS-PDMS for selfassembly with 12-nm pitch and the self-assembly and ordering of PS-PDMS along the guide line of hydrogen silsesquioxane (HSQ) resist made by EB drawing. We have discussed the possibility of achieving long-range ordering of $\sim 6-\mathrm{nm}$-sized dot arrays, with a pitch of about $10 \mathrm{~nm}$ between the guide lines.

\section{BCP selection and experimental process}

\subsection{BCP selection for $5 \mathrm{~Tb} / \mathrm{in}^{2}[8]$}

We selected the molecular weight (MW) of PS-PDMS based on the theoretical relationship between the product of $\chi$ (Flory-Huggins parameter) and $\mathrm{N}$ (total number of segments), i.e., $\chi \mathrm{N}$, and the PDMS concentration of BCP for fine spheres. Accordingly, we selected four different MW ranges of PS-PDMS, as shown in Fig. 1(a). For fabrication of fine nano-dots, we have to select proper PDMS volume fraction and $\chi \mathrm{N}$ surrounding boundaries between sphere region and cylinder structure region (dot line) and disordered region (broken line) by selecting molecular weight. As the self-assembling using 4 BCPs selected here, it could be observed that the self-assembled pitch and the dot size decrease with the $\chi \mathrm{N}$ (Fig. 1(b)). Using the different stages during the process of self-assembly, including annealing, and reactive ion etching (RIE), we could obtain very fine pitches of self-assembled PDMS dots, as shown in Fig. 2. It could be observed from Fig. 2 that average dots with pitches of $\sim 33$ and $12 \mathrm{~nm}$ were obtained by using PS-PDMS of MWs of 30000-7500 g/mol and 7000-1500 g/mol, respectively. Further, it could be realized that self-assembly with PS-PDMS of MW 7000- 
$1500 \mathrm{~g} / \mathrm{mol}$ is suitable for achieving $5 \mathrm{Tbit} / \mathrm{in}^{2}$, because the two-dimensional pitch is approximately $10 \mathrm{~nm} \times 12 \mathrm{~nm}$.

\subsection{Ordering process}

Ordering with graphoepitaxy could be achieved by using three types of guide templates, namely guide line, post lattice, and mixing patterns [7]. This study used one of these templates to carry out the self-assembled ordering of nanodots. The sequential experimental procedure is schematically shown in Fig. 3. As the first step, we designed the template pattern. Subsequently, the guide pattern was fabricated by $30-\mathrm{keV}-\mathrm{EB}$ drawing with HSQ negative resist. Following the process of developing, brushing treatment was carried out. This brushing treatment was done to imitate the EB-drawn resist pattern as a guide pattern for the ordering of self-assembled nanodots. The brushing material was PS of MW 4000 with a concentration of $2 \%$ in propylene glycol monomethyl ether acetate (PGMEA) solution. Then, the solution on the EB-drawn template pattern was spin coated at a revolution of $4000 \mathrm{rpm}$. To retain a monolayer of PS film, the spin coated sample was flushed with toluene. Finally, we selfassembled PS-PDMS BCP on the brushed sample by spin-coating and annealing, followed by two-step RIE with $\mathrm{CF}_{4}$ and $\mathrm{O}_{2}$ gas, as shown in Fig. 4. RIE allowed the ordering of selfassembled PDMS dot arrays by graphoepitaxy using the guide pattern.

\subsection{EB drawing [9]}

The EB drawing system used in this study consisted of a commercial high-resolution scanning electron microscope (SEM) (JSM6500F, JEOL Co.) and an EB-drawing controller (Beam Draw, Tokyo Technology Co.). The system has a large current probe with a small probe diameter at the maximum acceleration voltage of $30 \mathrm{kV}$. The $\mathrm{EB}$ drawing process is as follows.

The sample was prepared with a piece of Si of size $1 \mathrm{~cm} \times 1 \mathrm{~cm}$. The HSQ resist was coated by a spinner and prebaked at $110{ }^{\circ} \mathrm{C}$ for $10 \mathrm{~min}$. The thickness of the resist was $22-40$ $\mathrm{nm}$. The template pattern in the HSQ resist was EB-drawn at a probe current of $100 \mathrm{pA}$ and an acceleration voltage of $30 \mathrm{kV}$. The address resolution was $2.5 \mathrm{~nm} \times 2.5 \mathrm{~nm}$. After the EB drawing, we developed the sample using a developer composed of $2.4 \%$ tetramethylammonium hydroxide (TMAH) to obtain the template made of HSQ resist. The system has the ability to form nanometer-sized dot arrays with a pitch of 15-18 nm and a dot diameter of $<13 \mathrm{~nm}[10]$.

\subsection{Self-assembly of PS-PDMS}

Using the brushing-treated template, we self-assembled the PDMS dots on the template as follows. PS-PDMS BCPs with MW of 30000-7500 and 7000-1500 g/mol were used. A 
solution of PGMEA with 2\% PS-PDMS was prepared. After spin-coating the solution on the template, the sample was annealed at $170{ }^{\circ} \mathrm{C}$ for $11 \mathrm{~h}$. As a result, the PS-PDMS molecules were self-assembled by guiding with the lines or the mixing template. The PS-PDMS layer structure was estimated to be composed of three layers, namely PDMS top layer, PDMS dots in PS base layer, and PS bottom layer, in sequence from the top to the Si substrate [3]. In order to separate the self-assembled and ordered PDMS dot arrays from the PS base layer, two-step RIEs were carried out. To remove the PDMS top layer, $\mathrm{CF}_{4}$-RIEs of the samples were done for $10 \mathrm{~s}$ and $60 \mathrm{~s}$ using BCPs with MW of 30000-7500 and 7000-1500 g/mol, respectively. Subsequently, $\mathrm{O}_{2}$-RIEs were done for $180 \mathrm{~s}$ to remove the PS base and bottom layers between the PDMS dots [7]. The RIEs allowed the self-assembled PDMS dots ordering to appear. The etching corresponds to development in EB drawing.

\section{Experimental results and discussion}

\subsection{Guide pattern selection}

We measured the post size and pitch for ordering of the self-assembled dots to consider the optimal guide pattern for the samples. While, we require to fabricate optimal guide pattern with distances of the guide posts or lines as follows. (1) EB-drawn dot size for the post must be the same as that of self-assembled dot, and (2) the pitch is also the same as obtained with a few times of average self-assembled dot pitch with an error of a few $\mathrm{nm}$ in case of post-lattice guide. However, when the self-assembled dot attains the diameter $6 \mathrm{~nm}$, it becomes highly difficult to make very fine dots by EB drawing. However, we could not fabricate such a fine dot. Consequently, we used three types of templates with BCP of 30000-7500 g/mol MW, because the dot diameter is approximately $22 \mathrm{~nm}$. On the other hand, only guide-line template can be used when BCP of 7000-1500 g/mol MW is used, because the dots cannot be EBdrawn with a post size of $6 \mathrm{~nm}$.

\subsection{Ordering of self-assembled dots with BCPs of MW 30000-7500 and 7000-1500 g/mol}

We tried to order self-assembled PDMS dots with BCPs of MW 30000-7500 and 7000-1500 $\mathrm{g} / \mathrm{mol}$ using the mixing and guide-line templates, respectively.

In case of BCP of MW 30000-7500 g/mol, the template pattern with the line and postmixing guide templates was EB-drawn. The pattern was made of HSQ resist on Si substrate. After brushing PS on the template, we self-assembled the BCP to get ordered PDMS dot arrays along the guide line, as shown in Fig. 5. The post diameter slightly increased to become $\sim 27 \mathrm{~nm}$. Although the increase occurred by brushing, the self-assembly with the 
template ordered the dot arrays along the guide lines, because the pitch was in good agreement with that obtained by using pitch three times.

In case of BCP of MW 7000-1500 g/mol, we used guide-line template. Figure 6 shows the SEM images of self-assembled PDMS dot arrays ordered along the guide lines with 3 and 7 dot lines. The average space gaps between the guide lines are approximately 40 and $83 \mathrm{~nm}$. As a pitch of the ordered dot arrays is $\sim 10.9 \mathrm{~nm}$ driven from ordering property [11], the spaces are 3.67 and 7.55 times the pitch, respectively. The spaces are near 3.5 and 7.5. Considering the edge roughness of the guide line, the EB-drawn spaces are available for ordering the self-assembled BCP with a MW of 7000-1500 g/mol between the guide lines. Consequently, the designed space should be set at a middle between $n$-times and $(n+1)$-times pitches for $n$-dot array ordering between the guide lines.

\section{Conclusions}

In this study, we have studied the ordering of self-assembled nanodot arrays with a pitch of 12 $\mathrm{nm}$ and $33 \mathrm{~nm}$ for patterned media, using the mixing and guide-line templates made of EB resist on Si substrate. Based on the experimental results, we could arrive at the following conclusions:

1) We developed the process technology for self-assembled nanodot arrays with a pitch and a diameter of $12 \mathrm{~nm}$ and $6 \mathrm{~nm}$, respectively, ordered using the EB-drawn resist guide-lines.

2) We also demonstrated the possibility of ordering the self-assembled nanodots with the abovementioned dot diameter and pitch using the guide-line templates for ultrahighdensity patterned media with a density of approximately $5 \mathrm{~Tb} / \mathrm{in}^{2}$.

3) We ordered five nanodot lines along the guide lines between the lines.

4) The ordering of self-assembled BCP of MW 30000-7500 g/mol was achieved using the mixing template.

5) Fabrication process, 30-keV EB drawing, PS-brushing treatment, and self-assembly with PS-PDMS of MW 7000-1500 g/mol was developed for ordering dot arrays of diameter 6 $\mathrm{nm}$ with a pitch of about $10 \mathrm{~nm}$ between the guide lines.

\section{Acknowledgments}

This work was funded by the New Energy and Industrial Technology Development Organization (NEDO) under the "Development of nanobit technology for ultrahigh density magnetic recording (Green IT)" project, and also supported by JSPS KAKENHI Grant Number 24656435, and by JST Sentan Grant Number H24sentan181-25. 


\section{Reference}

1) I. Bita, J. Yang, Y. Jung, C. Ross, E. Thomas, K. Berggren, Science 321 (2008) 939-943.

2) J. G. Son, A. F. Hannon, K. W. Gotrik, A. Alexander-Katz, C. A. Ross, Adv. Mater. 23 (2011) 634-639.

3) J. Chang, J. G. Son, A. F. Hannon, A. Alexander-Katz, C. A. Ross, K. K. Berggren, ACS NANO 6 (2012) 2071-2077.

4) M. Sakurai, Y. Kamata, H. Hieda, Toshiba Rev. 57 (2002) 52-55.

5) C. T. Black, Nano 1 (2007) 147-150.

6) H. Kitano, S. Akasaka, T. Inoue, F. Chen, MM. Takenaka, H. Hasegawa, H. Yoshida, H. Nagano, Langmuir 23 (2007) 6404-6410.

7) S. Hosaka, T. Akahane, M Huda, T. Tamura, Y. Yin, N. Kihara, Y. Kamata, A. Kitsutsu, Microelectron. Eng. 88 (2011) 2571-2575.

8) M. Huda, J. Liu, Y. Yin, S. Hosaka, Jpn. J. Appl. Phys. 51 (2012) 06FF10 (1-5).

9) S. Hosaka, Y. Tanaka, M. Shirai, Z. Mohamad, Y. Yin, Jpn. J. Appl. Phys. 49 (2010) $046503(1-3)$.

10) T. Komori, H. Zhang, T. Akahane, Z. Mohamad, Y. Yin, S. Hosaka, Jpn. J. Appl. Phys. 51 (2012) 06FB02 (1-4).

11) S. Hosaka, T. Akahane, M. Huda, H. Zhang, Y. Yin, Applied Materials Interface (in press). 


\section{Figure Captions}

Fig. 1 Micro phase separation diagram and 4 PS-PDMSs with selected molecular weights and dot sizes of the PS-PDMS for the $\chi \mathrm{N}$, (a).the $\chi \mathrm{N}$ and nanostructures and (b) dot pitch and size for the $\chi \mathrm{N}$.

Fig. 2 SEM images of PDMS dots self-assembled without guide pattern using block copolymer PS-PDMS of molecular weight (a) 30000-7500 g/mol and (b) 7000-1500g/mol.

Fig. 3 Experimental process flow for ordering self-assembled PS-PDMS nanodots using guide template: (a) design of guide pattern, (b) EB drawing of the guide template, (c) brushing treatment on the guide template, (d) ordered self-assembly of PS-PDMS PDMS dots.

Fig. 4 Process flow for PS-PDMS self-assembly.

Fig. 5 Graphoepitaxy using guide-line and post-lattice mixing templates (SEM image of ordering of $33 \mathrm{~nm}$ pitched self-assembled dot arrays).

Fig. 6 SEM images of graphoepitaxy ordering of self-assembled PDMS dot arrays with a dot size of $6 \mathrm{~nm}$ and pitch of $12 \mathrm{~nm}$ between guide lines, (a) 3dot arrays with a gap of $40 \mathrm{~nm}$ and (b) 7 dot arrays with a gap of $83 \mathrm{~nm}$. 


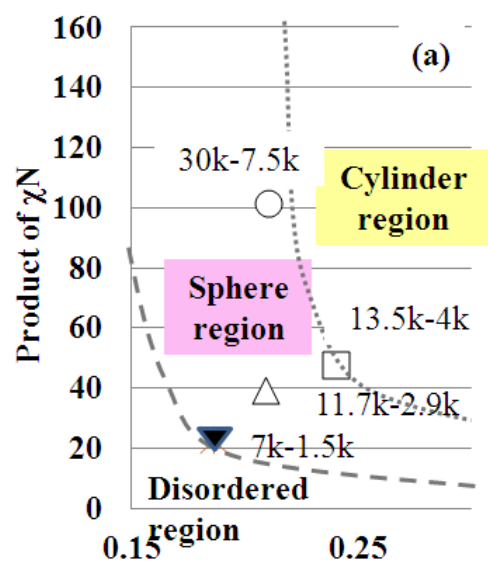

PDMS block volume fraction $\mathrm{f}_{\text {PDNS }}$

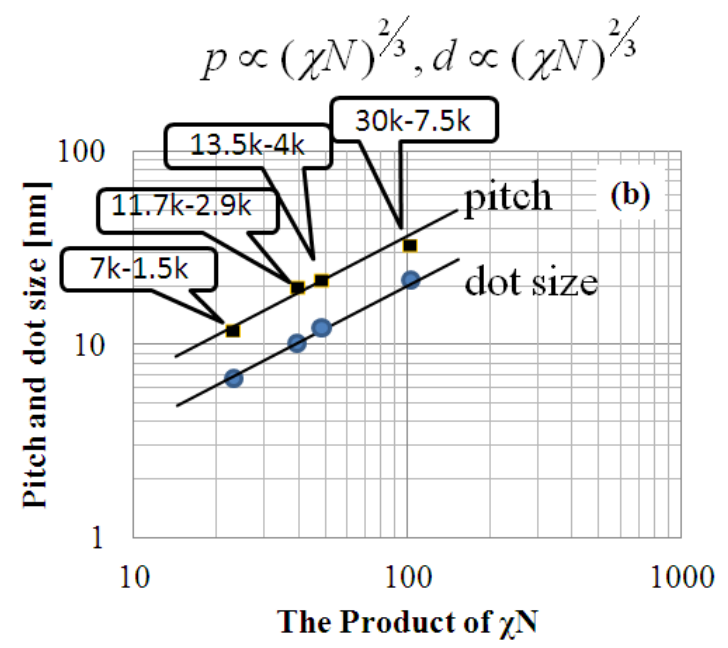

Fig. 1 

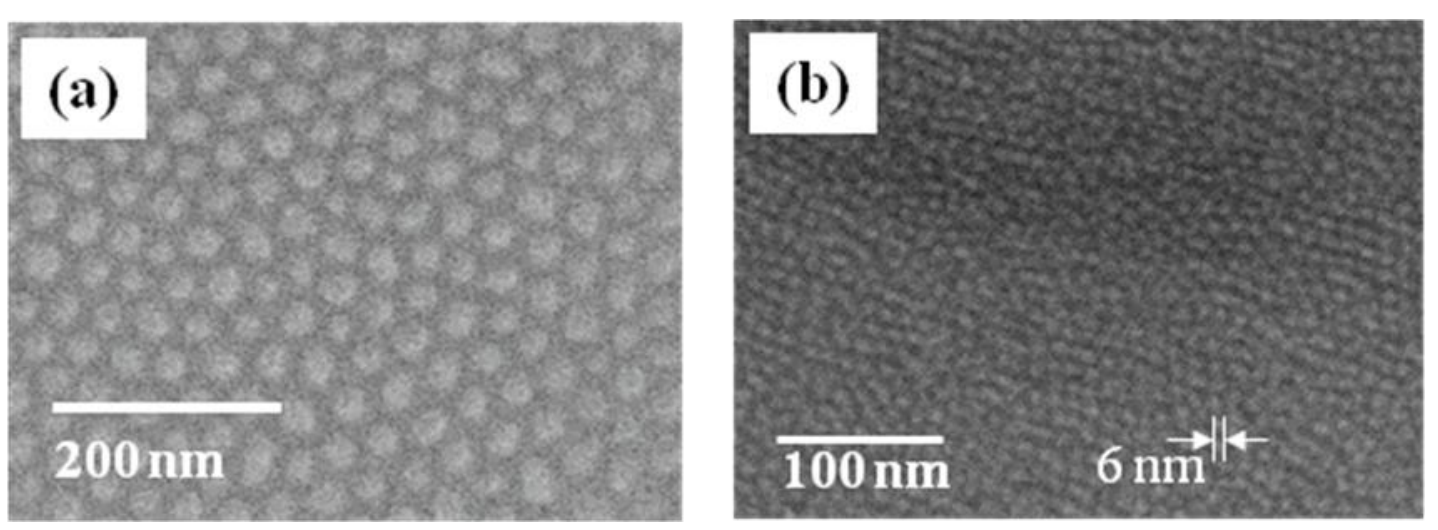

Fig. 2 


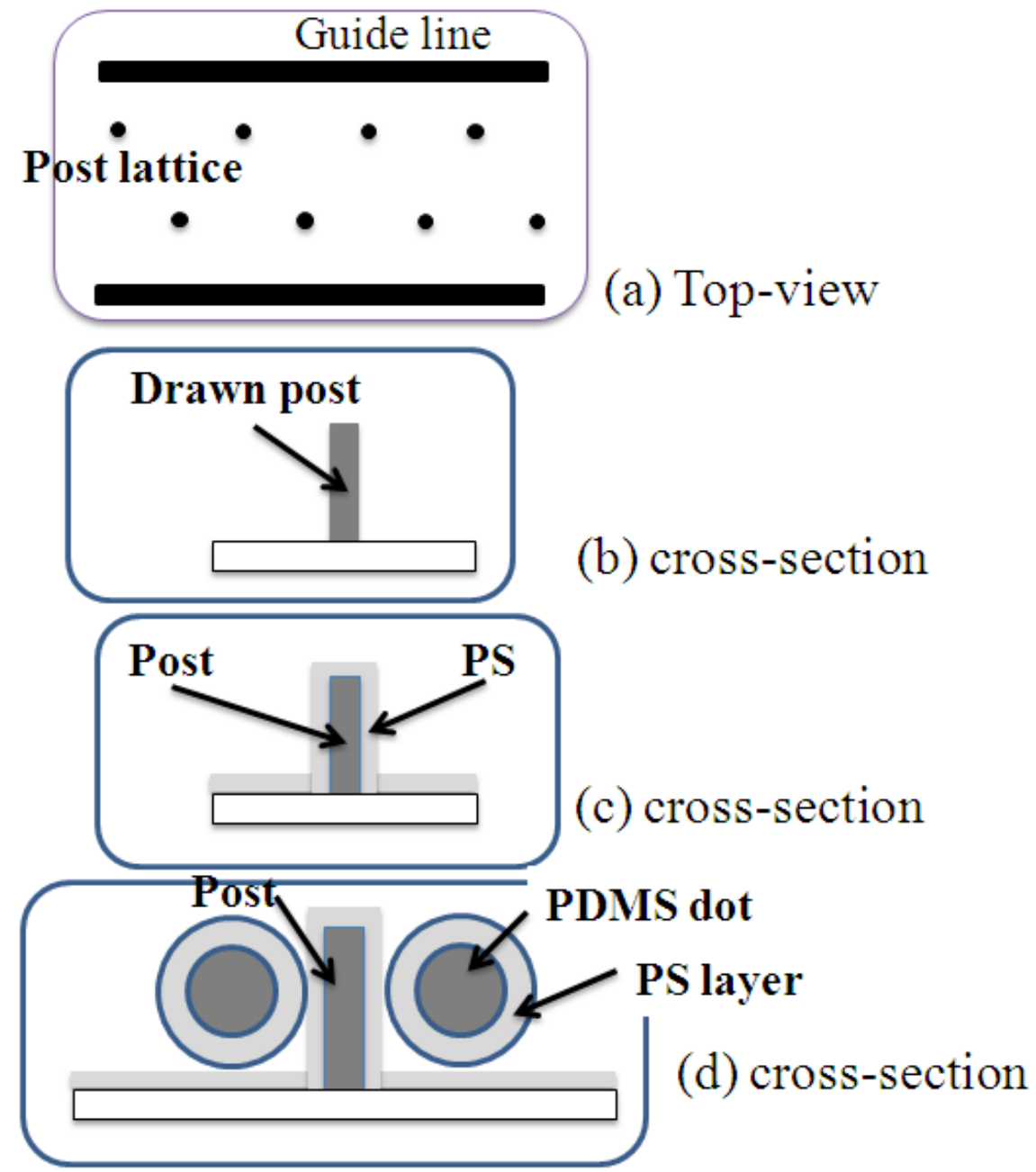

Fig. 3 


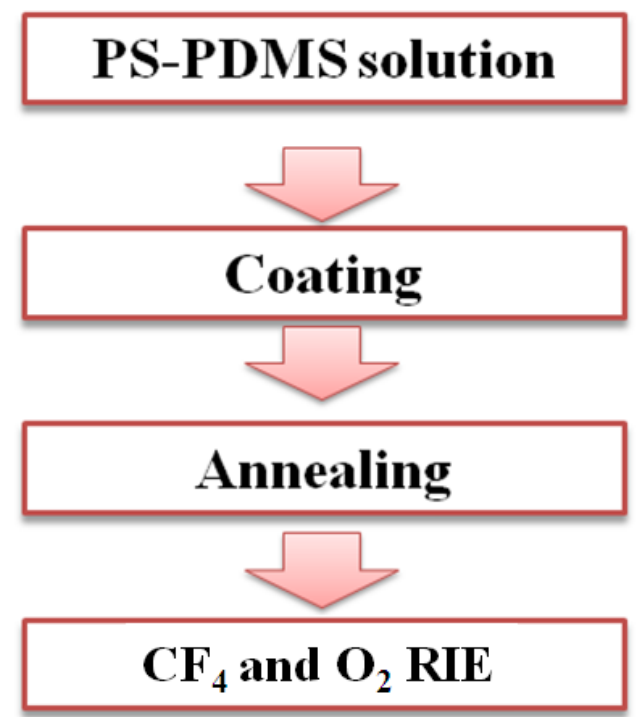

Fig. 4 
MNE Paper: S. Hosaka et al.

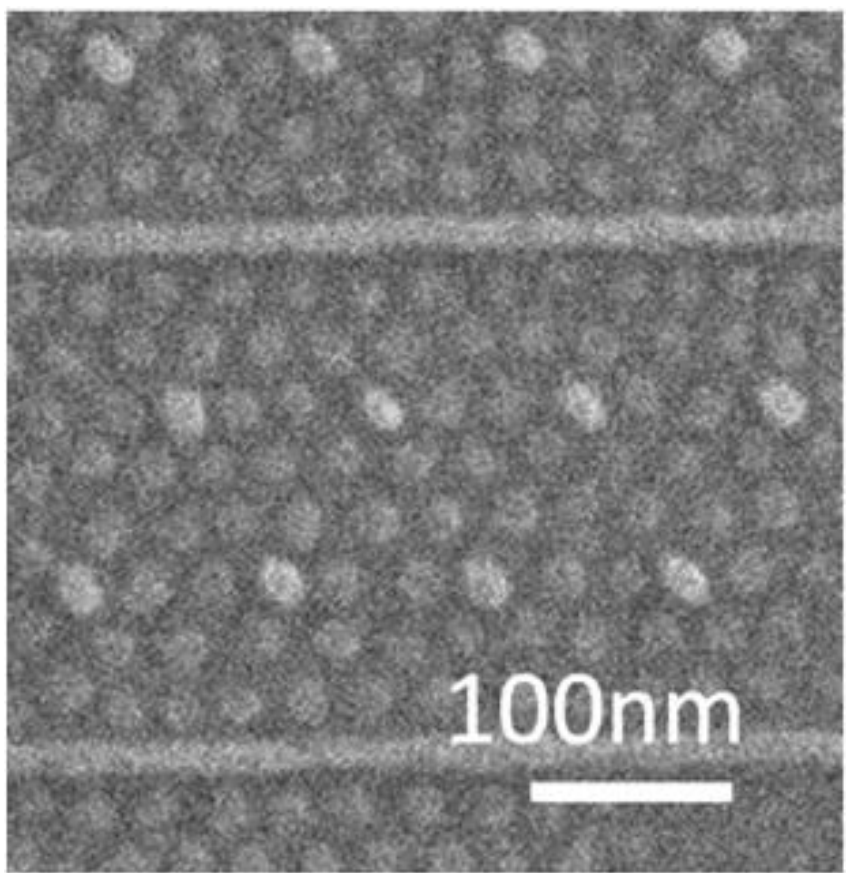

Fig. 5 
(a)
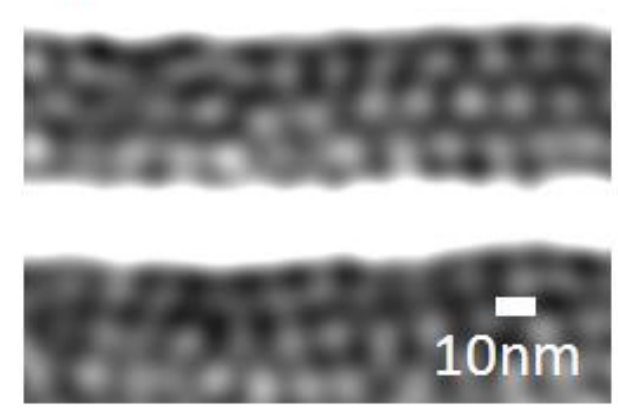

(b)

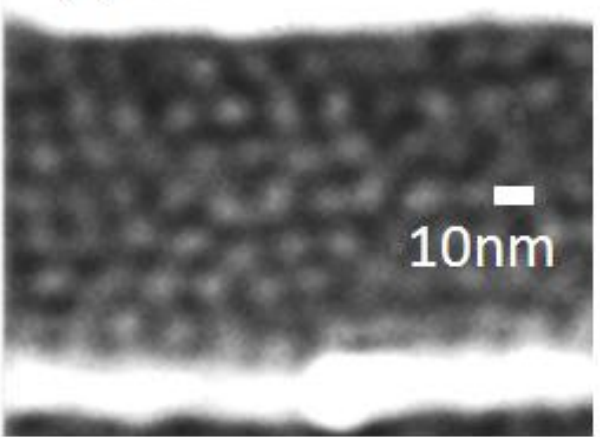

Fig. 6 
Ordering of 6-nm-sized nanodot arrays with 10-nm-pitch using self-assembled block copolymers along electron beam-drawn guide-lines

\author{
Sumio Hosaka ${ }^{1 *}$, Takashi Akahane ${ }^{1}$, Miftakhul Huda ${ }^{1}$, Takuya Komori ${ }^{1}$, You Yin ${ }^{1}$ \\ ${ }^{1}$ Graduate School of Engineering, Gunma University \\ 1-5-1 Tenjin-cho, Kiryu 376-8515, Japan \\ *Corresponding author: hosaka@el.gunma-u.ac.jp, Tel. +81-277-30-1721, Fax. \\ $+81-277-30-1707$
}

Graphical abstract

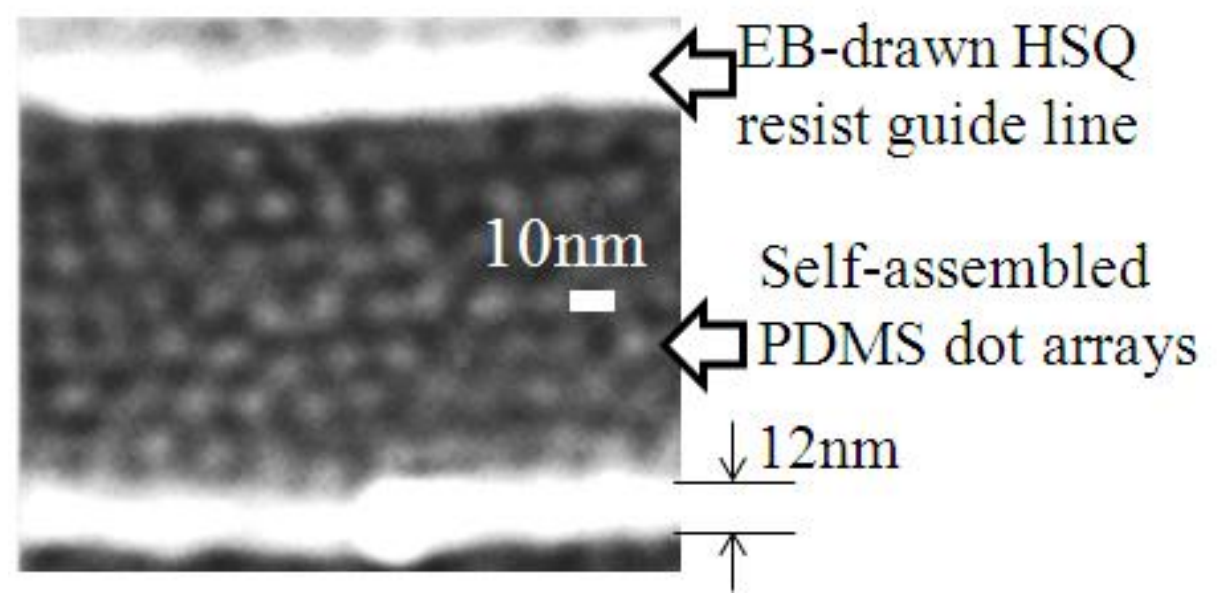

Fig 1 SEM image of 7-ordered PDMS dot arrays between EB-drawn fine guide lines by graphoepitaxy (self-assembly) with guide lines. 